\title{
DISCUSSION
}

Tinsley: On the correlation of morphological peculiarity with ultraviolet excess, I would like to mention a study that R. B. Larson and I made earlier this year. We studied the distribution of galaxies in Arp's "Atlas of Peculiar Galaxies" in the (U-B, B-V) two-colour plane. Interacting galaxies have an unusual colour distribution, in the sense that many have a strong ultraviolet excess. We interpreted the colour in terms of unusually high star formation rates, which are presumably a consequence of the interaction. Maybe Dr Heidmann is seeing a similar effect.

\section{CLUSTER MEMBERSHIP OF SEYFERT GALAXIES}

\author{
K. -H. Schmidt
}

I would like to comment on very preliminary results concerning the cluster membership of Seyfert galaxies. Two years ago van den Bergh stated in a discussion of the bright classical Seyfert galaxies that these objects were mainly field galaxies. We extend this discussion to about seventy objects in the list of Drs Khachikian and Weedman (Astrophys. J., 192, 581, 1974). By a cross correlation of this 1ist with Dr Abel1's catalogue of rich clusters taking account both of the positions in the sky and the distances of the objects, we find only two certain cluster members - the we11-known case NGC 1275 in the Perseus cluster and Markarian 298 in the Hercules cluster - and three probable or possible other cluster members. Taking into account the fact that only some fifty objects of Khachikian and Weedman fall within the distance range of the Abell catalogue, we find that only a few per cent of the Seyfert galaxies are members of Abe11 clusters. Within a factor of two, this percentage is the same as the percentage of cluster galaxies relative to all galaxies. Therefore, this result does not point to a pronounced field membership of the Seyfert galaxies.

Further, there seems to be a hint that Seyfert galaxies are found in clusters. Four out of five certain or probable members of Abel1 clusters are class 2 Seyferts. Although the statistics are as yet poor, this result is unexpected if one takes into account the fact that 53 of the 70 Seyferts in the 1ist of Khachikian and Weedman belong to class 1 and only 17 to type 2 .

\section{DISCUSSION}

Abel2: David Jenner and I found a Seyfert galaxy in the Virgo cluster. It is a norma1-appearing spiral, whose NGC number I have forgotten (off hand). We shall publish a note on it.

Huchra: When you compute probabilities from the cluster and Seyfert catalogue cross correlation, do you correct for the very different sky coverage of the catalogues? The majority of the Seyferts come from Markarian's first 1 ists which cover only a small region of the sky. 
Schmidt: Yes, I correct for this effect.

Ambartsumian: Have the clusters to which Seyfert galaxies belong any special peculiarities?

Schmidt: No, they are normal clusters.

\section{THE RESULTS OF OBSERVATIONS OF DOUBLE \\ GALAXIES IN THE UBV SYSTEM}

Aleksander Tomov

In the Astronomical Observatory of the town of Belogradchik a onechannel electrophotometer in the UBV system with pulse counting has been installed at the $60-\mathrm{cm}$ Cassegrain telescope. The photoamplifier used is of the type EMI 6256B (1). With this telescope the author has carried out observations of 80 double galaxies during the period 1974 to the middle of 1977 , using the differential method of observation with 3 diaphragms (24", 54" and 135") depending on its diameter.

The most important results are the correlation between the colour indices of individual components of double galaxies, Holmberg having obtained the correlation coefficient $R_{(B-V)}=0.80 \pm 0.06$ (2).

The author set himself the aim of checking the degree of correlation for the two colours $(B-V)$ and $(U-B)$ for individual classes of double galaxies; E-E and S-S - 60 double galaxies and E-S - 20 double galaxies.

The correlation coefficients $R$ for the 60 double galaxies ( $E-E$ and $\mathrm{S}-\mathrm{S})$ are $\mathrm{R}_{(\mathrm{B}-\mathrm{V})}=0.885 \pm 0.046$ and $\mathrm{R}_{1(\mathrm{U}-\mathrm{B})}=0.824 \pm 0.067$.

The correlation coefficients for 20 double galaxies (E-S) are $\mathrm{R}_{(\mathrm{B}-\mathrm{V})}=0.620 \pm 0.085$ and $\mathrm{R}_{\mathrm{l}(\mathrm{U}-\mathrm{B})}=0.512 \pm 0.098$.

In addition the author selected individual galaxies taken from reference (3) randomly together as double galaxies. It turned out that in this case, too, there is a correlation between the colour indices.

The correlation coefficients $R^{\prime}$ for 55 randomly selected pairs of $\mathrm{E}-\mathrm{E}$ and $\mathrm{S}-\mathrm{S}$ galaxies are

$$
\begin{aligned}
& R^{\prime}(B-V)=0.580 \pm 0.072 \\
& R_{l^{\prime}(U-B)}=0.382 \pm 0.085 .
\end{aligned}
$$

For $\mathrm{E}$ and $\mathrm{S}$ galaxies selected randomly, the 27 pairs have correlation coefficients

$$
\begin{aligned}
& R_{(B-V)}^{\prime}=0.125 \pm 0.090 \\
& R_{l^{\prime}(U-B)}=0.084 \pm 0.098
\end{aligned}
$$

